\title{
Trigonometric parallaxes of ten ultracool subdwarfs ${ }^{\star} \star \star$
}

\author{
E. Schilbach ${ }^{1}$, S. Röser ${ }^{1}$, and R.-D. Scholz ${ }^{2}$ \\ 1 Astronomisches Rechen-Institut, Zentrum für Astronomie der Universität Heidelberg, Mönchhofstraße 12-14, \\ 69120 Heidelberg, Germany \\ e-mail: [elena;roeser]@ari.uni-heidelberg.de \\ 2 Astrophysikalisches Institut Potsdam, An der Sternwarte 16, 14482 Potsdam, Germany \\ e-mail: rdscholz@aip.de
}

Received 3 November 2008 / Accepted 25 November 2008

\begin{abstract}
Aims. We measure absolute trigonometric parallaxes and proper motions with respect to many background galaxies for a sample of ten ultracool subdwarfs.

Methods. Observations were taken in the $H$-band with the OMEGA2000 camera on the $3.5 \mathrm{~m}$-telescope at Calar Alto, Spain during a time period of 3.5 years. For the first time, the reduction of the astrometric measurements was carried out directly with respect to background galaxies. We obtained absolute parallaxes with mean errors ranging between 1 and 3 mas.

Results. With six completely new parallaxes we more than doubled the number of benchmark ultracool (>sdM7) subdwarfs. Six stars in the $M_{K_{\mathrm{s}}}$ vs. $J-K_{\mathrm{s}}$ diagram fit perfectly to model subdwarf sequences from M7 to L4 with $[M / H]$ between -1.0 and -1.5 , whereas 4 are consistent with a moderately low metallicity $([M / H]=-0.5)$ from M7 to T6. All but one of our objects have large tangential velocities between 200 and $320 \mathrm{~km} \mathrm{~s}^{-1}$ typical of the Galactic halo population.

Our results are in good agreement with recent independent measurements for three of our targets and confirm the previously measured parallax and absolute magnitude $M_{K_{\mathrm{S}}}$ of the nearest and coolest (T-type) subdwarf 2MASS 0937+29 with higher accuracy.

For all targets, we also obtained infrared $J, H, K_{\mathrm{s}}$ photometry at a level of a few milli-magnitudes relative to 2MASS standards.
\end{abstract}

Key words. astrometry - stars: distances - stars: kinematics - stars: low-mass, brown dwarfs - subdwarfs - solar neighborhood

\section{Introduction}

Subdwarfs were originally defined by Kuiper (1939) as stars with spectral types A-K lying "not over 2-3 mag below the main sequence" in optical colour-magnitude diagrams. They are low-metallicity stars which typically have large space velocities. They are local representatives of the Galactic thick disk and halo populations and show up preferentially in high proper motion surveys. However, compared to thin disk stars with solar metallicities, subdwarfs are a rare species. In the well-investigated close solar neighbourhood $(d<10 \mathrm{pc})$ there are only 4 subdwarfs among the total of 348 objects with accurately measured trigonometric parallaxes (Henry et al. 2006).

The coolest subdwarfs with late-K and $\mathrm{M}$ spectral types were described about ten years ago by Gizis (1997). He developed a corresponding spectroscopic classification scheme separating normal M dwarfs with solar metallicities $([M / H] \approx 0.0)$ from $\mathrm{M}$ subdwarfs $(\mathrm{sdM})$ with metallicities $[M / H] \approx-1.2$ and extreme subdwarfs (esdM) with even lower metallicities $([M / H] \approx$ -2.0). The latest-type subdwarf classified by Gizis (1997) is the high proper motion star LHS 377 with a spectral type of sdM7.

\footnotetext{
* Based on observations collected with Omega 2000 at the $3.5 \mathrm{~m}$ telescope at the Centro Astronómico Hispano Alemán (CAHA) at Calar Alto, operated jointly by the Max-Planck Institut für Astronomie and the Instituto de Astrofísica de Andalucía (CSIC).

$\star \star$ Tables 1 and 2 are also available in electronic form at the CDS via anonymous ftp to

cdsarc.u-strasbg.fr $(130.79 .128 .5)$ or via

http://cdsweb.u-strasbg.fr/cgi-bin/qcat?J/A+A/493/L27
}

The search for very low-mass stars and brown dwarfs has experienced enormous progress in the last decade, mainly thanks to new deep optical and near-infrared (NIR) all-sky surveys, which also enable and support new high proper motion searches. Many objects even cooler than M dwarfs have been discovered; the new spectral types $\mathrm{L}$ and $\mathrm{T}$ have been invented to describe them (see Kirkpatrick 2005, and references therein), and their current census has reached nearly 700 objects (Gelino et al. 2008). A question of particular interest is the formation of low-mass stars and brown dwarfs in the low-metallicity regime. Relicts of the early Galaxy and the first generations of star formation may be detectable among the faintest objects in the local Galactic halo population. Interestingly, the new sky surveys have also revealed a new population of ultracool subdwarfs (hereafter UCSDs), i.e. metal-deficient stars and brown dwarfs of spectral types later than sdM7 extending into the new $\mathrm{L}$ and possibly $\mathrm{T}$ spectral classes (Lépine et al. 2003b,c; Scholz et al. 2004a,b; Sivarani et al. 2004; Burgasser et al. 2002, 2003a, 2004, 2007; Burgasser \& Kirkpatrick 2006; Lépine \& Scholz 2008).

Trigonometric parallax measurements of these new UCSDs are essential to determine their absolute brightnesses, effective temperatures and space motions. The first (sub-)stellar UCSDs with accurate distance estimates will serve as benchmark sources for our understanding of this new population and for their detailed classification. A new classification scheme for UCSDs is still under debate (Gizis \& Harvin 2006; Burgasser et al. 2007). Recently, Lépine et al. (2007) have revised the Gizis (1997) scheme for $\mathrm{M}$ subdwarfs and introduced a third class, the socalled ultrasubdwarfs (usdM), for the lowest metallicities. In 
Table 1. Targets with previously known proper motions, $J H K_{\mathrm{s}}$ photometry and spectral types.

\begin{tabular}{lccccc}
\hline \hline Name & $J$ & $\begin{array}{c}H \\
\text { (2MASS) }\end{array}$ & $K_{\mathrm{s}}$ & SpType & Ref. \\
& $(2)$ & $(3)$ & $(4)$ & $(5)$ & $(6)$ \\
\hline 2MASS 0532+8246 & 15.179 & 14.904 & 14.918 & sdL7 & $5 ; 5,11$ \\
2MASS 0937+2931 & 14.648 & 14.703 & 15.267 & d/sdT6 & $7 ; 1,11$ \\
SSSPM 1013-1356 & 14.621 & 14.382 & 14.398 & sdM9.5 & $3 ; 3,11$ \\
SSSPM 1256-1408 & 14.011 & 13.618 & 13.444 & & $12 ;-$ \\
SDSS 1256-0224 & 16.099 & 15.792 & 15.439 & sdL4: & $12 ; 4,11$ \\
LSR 1425+7102 & 14.775 & 14.405 & 14.328 & sdM8 & $12 ; 8,11$ \\
SSSPM 1444-2019 & 12.546 & 12.142 & 11.933 & d/sdM9 & $10 ; 10,11$ \\
LSR 1610-0040 & 12.911 & 12.302 & 12.019 & d/sdM7: $:^{\dagger}$ & $12 ; 2,11$ \\
2MASS 1626+3925 & 14.435 & 14.533 & 14.466 & sdL4 & $6 ; 6,11$ \\
LSR 2036+5059* & 13.611 & 13.160 & 12.936 & sdM7.5 & $12 ; 9,11$ \\
\hline
\end{tabular}

References: (Col. 6, first index for proper motion, others for sp. type): 1 - Burgasser et al. (2002); 2 - Lépine et al. (2003c); 3 - Scholz et al. (2004a); 4 - Sivarani et al. (2004); 5 - Burgasser et al. (2003a); 6 - Burgasser (2004); 7 - Vrba et al. (2004); 8 - Lépine et al. (2003b); 9 - Lépine et al. (2003a); 10 - Scholz et al. (2004b); 11 - Burgasser et al. (2007); 12 - Scholz et al. (unpublished, preliminary proper motion solution based on available SSS and 2MASS data).

$\dagger$ - Discovered by Lépine et al. (2003c) as the first possible L subdwarf. Cushing \& Vacca (2006) described it as a very peculiar object (M6p/sdM), and Dahn et al. (2008) recently found it to be an astrometric binary of the Galactic halo population consisting of a mildly metal-poor M dwarf and a substellar companion.

^ - Lépine et al. (2002) listed an erroneous position and proper motion for this object.

contrast, Jao et al. (2008) have considered novel methods for assigning spectral types from K3 to M6 dwarfs, discrediting the previous subdwarf metallicity classes and suggesting instead a more complex investigation of temperature, metallicity and gravity features. Such a three-dimensional scheme (temperature/clouds, metallicity, gravity) has also been proposed by Kirkpatrick (2005) for late-M, L and T dwarfs. However, the numbers of well-investigated subdwarfs are still too small (see Burgasser et al. 2007, for an overview) to fill the required grid of subtypes with benchmark sources.

\section{Target selection}

In 2004, when we initiated our subdwarf parallax programme, there were only nine UCSDs known, and all happened to be visible from a northern telesope site like Calar Alto. Among them, there were five M-type objects originally detected in optical high proper motion surveys by Lépine and co-workers (LSR 1425+7102, LSR 1610-0040, LSR 2036+5059) and Scholz and co-workers (SSSPM 1013-1356, SSSPM 1444-2019), three L- and T-type objects originally detected in the NIR Two Micron All Sky Survey (2MASS; Skrutskie et al. 2006) by Burgasser and co-workers (2MASS 0532+8246, 2MASS 0937+2931, 2MASS 1626+3925), and one object detected in the spectroscopic database of the deep optical Sloan Digital Sky Survey (SDSS; York et al. 2000) by Sivarani et al. (2004). The 2MASS photometry as well as spectral types from different sources are given in Table 1. We have included one more object (SSSPM 1256-1408), also detected in the high proper motion survey by Scholz et al. (unpublished) using the SuperCOSMOS Sky Surveys (SSS) data (Hambly et al. 2001), which is still lacking a spectral type. Its large optical-to-NIR $(R-J=+4.8 ; R$ from
SSS $)$ and small NIR $\left(J-K_{\mathrm{s}}=+0.57\right)$ colour indices are however typical of late-M UCSDs.

When we started our observations, only one of our targets (2MASS 0937+2931) had a preliminary trigonometric parallax measurement by Vrba et al. (2004). Meanwhile, there are three more parallaxes, all published in 2008, for 2MASS $0532+8246$ (Burgasser et al. 2008), LSR 1425+7102 and LSR 1610-0040 (Dahn et al. 2008).

\section{Observations and data reduction}

The observations were made with the OMEGA2000 camera on the $3.5 \mathrm{~m}$-telescope of the Centro Astronómico Hispano Alemán (CAHA) at Calar Alto, Spain. OMEGA2000 is a primefocus, near-infrared, wide-field camera that uses a $2 \mathrm{k} \times 2 \mathrm{k}$ HAWAII-2 focal plane array with a sensitivity from the $z$ to the $K$ band. The optics of the camera consists of a cryogenic focal reducer providing a $15.4^{\prime} \times 15.4^{\prime}$ field of view with a resolution of $0.45^{\prime \prime} /$ pixel. The astrometric observations were all obtained in the $H$-band. In all cases, at each epoch, we took 16 individual exposures of $60 \mathrm{~s}$ each (frames), with small offsets of a few arcseconds, thus totalling $16 \mathrm{mn}$ exposure time per object and night. The observations were taken between January 2005 and June 2008. The maximum epoch difference per target ranges from 3.1 to 3.4 years, with the number of useful epochs (nights) from 17 to 26. In addition, one observation (i.e., 16 individual frames) in the $J$ and $K_{\mathrm{s}}$ bands has been taken for each target.

Object detection and centroiding was carried out by use of the SEXTRACTOR software (Bertin \& Arnouts 1996). For the photometric reduction, standard stars are taken from 2MASS. On average, 100 stars per field with an accuracy better than $0.1 \mathrm{mag}$ in $2 \mathrm{MASS}$ were used as a reference. In a given photometric band, each of 16 frames has been reduced to the 2MASS photometric system, separately. As a rule, a linear fit was sufficient for stars fainter than 9th mag. For each object in a field, the final $J$-, $H$,- and $K_{\mathrm{s}}$-magnitudes were computed as averages of 16 values. The limiting magnitudes slightly vary from field to field and they reach $J=19, H=18, K_{\mathrm{s}}=17.5$, at least.

For each target, the astrometric data reduction was performed in several steps. At first, an appropriate "reference" frame was chosen. Each frame was reduced to the "reference" frame using a classical 2nd-order polynomial fit. As reference points for the "frame-to-frame" reduction we use anonymous field stars with $H$ magnitudes between 14 and 16.5; the number of reference stars varied between 75 for target 2MASS $0937+29$ and 1250 for LSR 2036+5059. The "reference" frame was transformed to an intermediate equatorial reference system defined by 2MASS stars in a given sky area. Again, a 2nd-order plate fit was carried out. The number of reference stars from 2MASS varied between 85 and 1650, the latter in the field around LSR $2036+5059$.

In this intermediate system, mean positions, proper motions and parallax were obtained via a rigorous single least-squares fit to the 5 unknowns, coupling the observational equations in RA and Dec via the parallax factor. Although mathematically correct, the solution may suffer from a relatively short time baseline and a non-uniform distribution of observations. Thus correlations may influence the results for proper motions and parallax. In order to check the robustness of the solutions, two additional least-squares procedures were carried out. The first of these treated the equations in RA and Dec separately, yielding two solutions for the parallax. In this check, correlations between RA and Dec. are prohibited. Evidently, the formal 
Table 2. Absolute parallaxes ( $\pi(\mathrm{abs}))$, absolute proper motions $\left(\mu_{\alpha} \cos \delta, \mu_{\delta}\right)$, and infrared magnitudes $\left(J, H, K_{\mathrm{s}}\right)$ of ultracool Subdwarfs.

\begin{tabular}{|c|c|c|c|c|c|c|c|c|c|c|c|}
\hline Name & $\begin{array}{c}\text { RA J2000.0 } \\
{[\mathrm{h}]}\end{array}$ & $\begin{array}{c}\text { Dec J2000.0 } \\
\text { [deg] }\end{array}$ & $\begin{array}{c}\pi(\mathrm{abs}) \\
{[\mathrm{mas}]}\end{array}$ & $\begin{array}{c}\Delta_{\pi} \\
\text { [mas] }\end{array}$ & $\begin{array}{r}\mu_{\alpha} \cos \delta \\
{[\mathrm{mas} / \mathrm{yr}]}\end{array}$ & $\begin{array}{c}\mu_{\delta} \\
{[\mathrm{mas} / \mathrm{yr}]}\end{array}$ & $\begin{array}{c}J \\
{[\mathrm{mmag}]}\end{array}$ & $\begin{array}{c}H \\
{[\mathrm{mmag}]}\end{array}$ & $\begin{array}{c}K_{\mathrm{s}} \\
{[\mathrm{mmag}]}\end{array}$ & $\begin{array}{c}M_{K_{\mathrm{s}}} \\
{[\mathrm{mag}]}\end{array}$ & $\begin{array}{r}V_{t(\mathrm{LSR})} \\
{\left[\mathrm{km} \mathrm{s}^{-1}\right]}\end{array}$ \\
\hline \multirow[t]{2}{*}{ 2MASS 0532+82 } & 5.548452 & 82.779208 & 42.28 & -3.36 & 2039.46 & -1661.79 & 15145 & 14894 & 14904 & 13.03 & 289 \\
\hline & & & \pm 1.76 & \pm 1.37 & \pm 1.52 & \pm 1.64 & \pm 8 & \pm 5 & \pm 15 & \pm 0.09 & \pm 12 \\
\hline \multirow[t]{2}{*}{ 2MASS $0937+29$} & 9.626350 & 29.528189 & 163.39 & -3.39 & 944.15 & -1319.78 & 14622 & 14677 & 15407 & 16.47 & 53 \\
\hline & & & \pm 1.76 & \pm 1.18 & \pm 1.24 & \pm 1.21 & \pm 4 & \pm 7 & \pm 14 & \pm 0.03 & \pm 1 \\
\hline \multirow[t]{2}{*}{ SSSPM 1013-13 } & 10.218708 & -13.939245 & 20.28 & -5.11 & 69.44 & -1028.93 & 14637 & 14372 & 14303 & 10.84 & 241 \\
\hline & & & \pm 1.96 & \pm 1.24 & \pm 1.20 & \pm 1.33 & \pm 7 & \pm 5 & \pm 7 & \pm 0.21 & \pm 23 \\
\hline \multirow[t]{2}{*}{ SSSPM 1256-14 } & 12.937228 & -14.144533 & 18.76 & -0.38 & -741.11 & -1002.13 & 14040 & 13624 & 13458 & 9.82 & 305 \\
\hline & & & \pm 1.85 & \pm 1.10 & \pm 1.40 & \pm 1.38 & \pm 4 & \pm 5 & \pm 5 & \pm 0.21 & \pm 31 \\
\hline \multirow[t]{2}{*}{ SDSS 1256-02 } & 12.943648 & -2.414587 & 11.10 & -0.43 & -512.09 & -297.71 & 16157 & 16060 & 16061 & 11.29 & 242 \\
\hline & & & \pm 2.88 & \pm 1.11 & \pm 1.90 & \pm 1.79 & \pm 13 & \pm 8 & \pm 22 & \pm 0.56 & \pm 66 \\
\hline \multirow[t]{2}{*}{ LSR 1425+7102 } & 14.418059 & 71.035998 & 12.19 & -0.73 & -602.38 & -177.71 & 14828 & 14412 & 14245 & 9.68 & 240 \\
\hline & & & \pm 1.07 & \pm 0.67 & \pm 0.98 & \pm 0.99 & \pm 7 & \pm 8 & \pm 8 & \pm 0.19 & \pm 21 \\
\hline \multirow{2}{*}{ SSSPM 1444-20 } & 14.738983 & -20.323730 & 61.67 & -2.41 & -2906.15 & -1963.12 & 12602 & 12149 & 11952 & 10.90 & 261 \\
\hline & & & \pm 2.12 & \pm 1.48 & \pm 2.41 & \pm 2.71 & \pm 6 & \pm 4 & \pm 4 & \pm 0.07 & \pm 9 \\
\hline \multirow[t]{2}{*}{ LSR 1610-0040 } & 16.174711 & -0.681642 & 33.10 & -2.63 & -773.84 & -1231.58 & 12872 & 12304 & 12004 & 9.60 & 205 \\
\hline & & & \pm 1.32 & \pm 0.95 & \pm 0.91 & \pm 0.88 & \pm 7 & \pm 2 & \pm 5 & \pm 0.09 & \pm 8 \\
\hline \multirow[t]{2}{*}{ 2MASS $1626+39$} & 16.438927 & 39.422076 & 29.85 & -1.10 & -1374.14 & 238.01 & 14426 & 14464 & 14464 & 11.84 & 219 \\
\hline & & & \pm 1.08 & \pm 0.48 & \pm 0.96 & \pm 0.87 & \pm 5 & \pm 9 & \pm 10 & \pm 0.08 & \pm 8 \\
\hline \multirow[t]{2}{*}{ LSR 2036+5059 } & 20.606002 & 51.001279 & 21.60 & -1.00 & 751.93 & 1252.22 & 13628 & 13232 & 12969 & 9.64 & 311 \\
\hline & & & \pm 1.26 & \pm 1.13 & \pm 1.10 & \pm 1.31 & \pm 9 & \pm 5 & \pm 20 & \pm 0.13 & \pm 19 \\
\hline
\end{tabular}

accuracy of the parallax is always better from RA than from Dec. Therefore, a weighted mean parallax was computed. The second approach is an iterative one. At first, only proper motions are computed. The solutions are taken to remove the proper motion effect from the observed image displacements. From the residuals, the parallax (and mean position again) is determined from the coupled equations. The iterations start with the removal of the parallax effect from the original observations, and determining proper motions from the latter. The procedure converged after 2 to 3 iterations. In this check, correlations between proper motions and parallax are prohibited. For all ten targets, the three solutions for the parallaxes differed by less than $40 \%$ of their combined mean error. Therefore, we consider our rigorous solution as reliable, stable and robust.

Because of the deep observations and the large field of $15.4^{\prime} \times 15.4^{\prime}$, an appropriate number of galaxies was found in each field which were used to reduce the relative parallaxes and relative proper motions to absolute ones. Putting to zero the apparent parallaxes and proper motions of the galaxies yields the corrections converting relative parallaxes and relative proper motions to absolute ones for all other objects in the field. The images of all galaxies in the fields were visually inspected to select sufficiently compact and well measured reference objects. The number of useful galaxies varied between 12 in the case of 2MASS $0532+82$ to 105 in the case of SSSPM 1256-14.

\section{Results and discussion}

Table 2 compiles the astrometric and photometric results for the 10 targets. The first column contains the target's name. Columns 2 and 3 give the mean (barycentric) position (RA and Dec) of the target for equinox and epoch J2000.0. Columns 4 and 5 present the absolute parallaxes $\pi(\mathrm{abs})$ and the applied corrections $\Delta_{\pi}$ converting the relative parallaxes to absolute ones as $\pi(\mathrm{abs})=\pi(\mathrm{rel})-\Delta_{\pi}$, while Cols. 6 and 7 give the derived absolute proper motions of the targets. Columns 8-10 summarize the results of our photometric measurements. Column 11 gives the absolute magnitude $M_{K_{\mathrm{s}}}$ computed from the corresponding trigonometric parallax and $K_{\mathrm{s}}$, whereas the last column lists the tangential velocities corrected for solar motion.
Four of our targets have already published trigonometric parallaxes. These are 2 MASS $0532+82$ with $\pi=37.5 \pm 1.7$ mas $\left(M_{K_{\mathrm{s}}}=12.79 \pm 0.18 \mathrm{mag}\right)$ by Burgasser et al. (2008), 2MASS $0937+29$ with $\pi=162.84 \pm 3.88$ mas $\left(M_{K_{\mathrm{s}}}=16.46 \pm 0.14 \mathrm{mag}\right)$ by Vrba et al. (2004), LSR $1425+7102$ and LSR 1610-0040 with, respectively, $\pi=13.37 \pm 0.51 \mathrm{mas}\left(M_{K_{\mathrm{s}}}=9.97 \pm 0.13 \mathrm{mag}\right)$ and $\pi=31.02 \pm 0.26$ mas $\left(M_{K_{\mathrm{s}}}=9.48 \pm 0.04 \mathrm{mag}\right)$ both by Dahn et al. (2008). All these parallaxes are based on observations with the $1.55 \mathrm{~m}$ Strand Astrometric Reflector at the USNO Flagstaff Station. The number of reference stars used varied between 6 and 16. Photometric data were taken mainly from the 2MASS Catalog. In spite of the different observation and reduction techniques, the published parallaxes coincide reasonably well with our results, i.e. the differences between two corresponding values are smaller than 2 times their mean errors. The parallax solutions from the observations with the $1.55 \mathrm{~m}$ Strand Astrometric Reflector take advantage of a better scale $\left(\approx 0.35^{\prime \prime} /\right.$ pixel versus $\approx 0.45^{\prime \prime} /$ pixel $)$, and, for LSR $1425+7102$ and LSR 1610-0040, of considerably larger ranges of epochs and numbers of nights. On the other hand, we benefit from a better SNR for the fainter targets, especially for photometry where the 2MASS Catalog is almost at its limit. Moreover, a relatively wide and deep field allows to use a large number of reference stars and, for the first time, to derive parallaxes directly with respect to galaxies and, consequently, to reduce any possible systematic bias in the parallax determination.

From the tangential velocities we infer that all objects except 2 MASS $0937+29$ exhibit halo kinematics. Postponing the discussion of the latter, we find that the remaining targets split into two groups in the CMD of Fig. 1. The first group is related to metallicity $[M / H]=-0.5$ in the Baraffe et al. (1997) isochrones for metal-poor low-mass stars, and is occupied by the objects LSR 1610-0040, SSSPM 1444-20 and 2MASS 0532+82. Ordered by luminosity they were classified as d/sdM7, d/sdM9 and sdL7 in Burgasser et al. (2007), respectively. For the first two objects, this is consistent with the Gizis (1997) classification scheme that rates dwarfs "d" with $[M / H] \approx 0$, and subdwarfs "sd" with $[M / H] \approx-1.2$. Note that 2 MASS $0532+82$, extensively discussed by Burgasser et al. (2008) perfectly fits the locus of $[M / H]=-0.5$, and therefore is not a real subdwarf according 


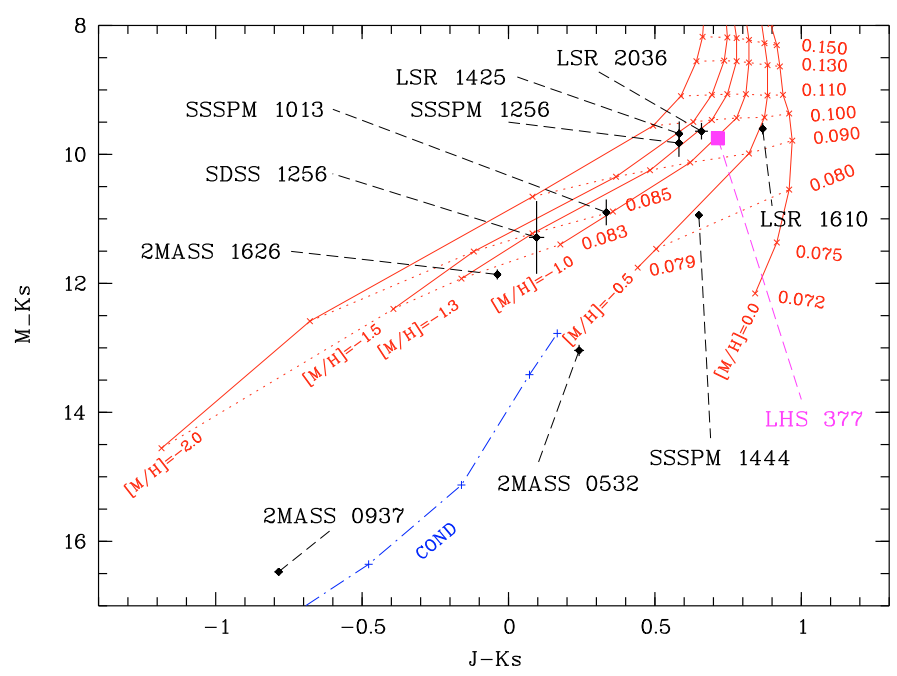

Fig. 1. Colour-absolute magnitude diagram $M_{K_{\mathrm{s}}}$ versus $J-K_{\mathrm{s}}$ containing our 10 targets and the sdM7 LHS 377 (filled square), a late type subdwarf with an already measured parallax (Monet et al. 1992; Gizis 1997), plotted here for completeness. The error bars correspond to $1 \sigma$ errors. The full (red) lines represent the absolute magnitudes and colours derived from the theoretical models by Baraffe et al. (1997) for $10 \mathrm{Gyr}$ old objects with $[M / H]$ from -2.0 to -1.0 , and by Baraffe et al. (1998) for 5 Gyr old objects with $[M / H]$ from -0.5 to zero. The model data points were converted from the CTI photometric system to the 2MASS system using the Carpenter (2001) transformations. Dotted lines are the lines of equal mass. The pluses connected by the dasheddotted line show COND model points for 5 Gyr old objects with less than 0.075 solar masses (Baraffe et al. 2003).

to the Gizis (1997) classification. Its moderately low metallicity has also been suggested by Scholz et al. (2004b) based on comparison with model colours $(I-J$ and $J-K)$. Therefore, we think these three objects can be used as benchmarks for the type "d/sd" with $[M / H]=-0.5$ from M7 to L7.

Of the remaining 6 objects, only one has a previously determined trigometric parallax, LSR $1425+7102$, measured by Dahn et al. (2008), and classified as sdM8 by Burgasser et al. (2007). The sdM7 LHS 377, which was not on our target list, is a close neighbour to LSR $1425+7102$ in the CMD. All our 6 objects populate the area between $[M / H]=-1.0$ and $[M / H]=-1.5$ in the Baraffe et al. (1997) isochrones. All are classified as "sd" by Burgasser et al. (2007) except our newly detected object SSSPM 1256-14, which we would classify as sdM8 based on its position in the CMD. The coolest object, the sdL4 2MASS 1626+39 has $M / M_{\odot}=0.083$ and $T_{\text {eff }}=2300 \mathrm{~K}$ when compared with the Baraffe et al. (1997) isochrones for $[M / H]=-1.0$. Our faintest (by apparent magnitude) target SDSS 1256-02 had the $K_{\mathrm{s}}$ magnitude in 2MASS given with a problem flag. Its 2MASS colour of $J-K_{\mathrm{s}}=0.66$ changes to 0.09 according to our photometry, and hence its metallicity in the Baraffe et al. (1997) models changes from -0.5 to -1.3 .

Our 6 targets as well as LHS 377 serve as benchmarks for the subdwarf population between M7 (LHS 377) and L4 (2MASS $1626+39)$ in the metallicity range between $[M / H]=-1.0$ and -1.5 . Baraffe et al. (2003) recently published new evolutionary models for the coolest brown dwarfs (T dwarfs), which they refer to as the COND models. In these models, dust opacity in the radiative transfer equation is neglected. The COND isochrone is shown in Fig. 1 only for objects with $M / M_{\odot}<0.075$. For masses up to $M / M_{\odot}=0.08$ it formally coincides with the $[M / H]=$ -0.5 isochrone. So, our targets from the $[M / H]=-0.5$ group have loci close to the COND isochrone, but the targets from the $[M / H]=-1.3$ class lie significantly above this isochrone.

2MASS $0937+29$ may be the faintest object along the extrapolated $[M / H]=-0.5$ isochrone in Fig. 1. It was also characterised as slightly metal-poor $(-0.4<[M / H]<-0.1)$ by Burgasser et al. (2003b; 2006) using spectral model comparisons. Compared to the 2MASS colour, our new photometry lead to a bluer $J-K_{\mathrm{s}}$, which again supports a sub-solar metallicity if we compare its location in Fig. 1 with the COND model points. Its relatively low tangential velocity $V_{t(\mathrm{LSR})}$ of about $50 \mathrm{~km} \mathrm{~s}^{-1}$ does not, however, reject a higher spatial velocity of 2MASS $0937+29$ with respect to the local standard of rest (LSR). The presently unknown $V_{\text {rad }}$ of 2 MASS $0937+29$ contributes to its space velocity components as $(U, V, W)_{\mathrm{LSR}}=$ (38-0.64 $\left.V_{\text {rad }},-30-0.21 V_{\text {rad }},+0.74 V_{\text {rad }}\right)$. Therefore, as long as the radial velocity of 2 MASS $0937+29$ is unknown, one cannot exclude the possibility that 2 MASS $0937+29$ is a member of the thick disk or even of the halo population.

In summary, we have measured infrared trigonometric parallaxes of ten ultracool subdwarfs, six of which for the first time. Absolute parallaxes have been determined with respect to galaxies, also for the first time. Compared to theoretical models, 4 stars have moderately low metallicity, $[M / H] \approx-0.5$, whereas 6 are consistent with $[M / H]$ between -1.0 and -1.5 . Nine out of ten definitely show halo kinematics from their tangential velocities, while 2 MASS $0937+29$ needs a large radial velocity to be kinematically excluded as a member of the disk.

\section{References}

Baraffe, I., Chabrier, G., Allard, F., \& Hauschildt, P. H. 1997, A\&A, 327, 1054 Baraffe, I., Chabrier, G., Allard, F., \& Hauschildt, P. H. 1998, A\&A, 337, 403 Baraffe, I., Chabrier, G., Barman, T. S., Allard, F., \& Hauschildt, P. H. 2003, A\&A, 402, 701

Bertin, E., \& Arnouts, S. 1996, A\&AS, 117, 393

Burgasser, A. J. 2004, ApJ, 614, L73

Burgasser, A. J., \& Kirkpatrick, J. D. 2006, ApJ, 645, 1485

Burgasser, A. J., Kirkpatrick, J. D., Brown, M. E., et al. 2002, ApJ, 564, 421

Burgasser, A. J., Kirkpatrick, J. D., Burrows, A., et al. 2003a, ApJ, 592, 1186 Burgasser, A. J., Kirkpatrick, J. D., Liebert, J., \& Burrows, A. 2003b, ApJ, 594, 510

Burgasser, A. J., Kirkpatrick, J. D., \& Burrows, A. 2006, ApJ, 639, 1095 Burgasser, A. J., Cruz, K. L., \& Kirkpatrick, J. D. 2007, ApJ, 657, 494 Burgasser, A. J., Vrba, F. J., Lépine, S., et al. 2008, ApJ, 672, 1159 Carpenter, J. M. 2001, AJ, 121, 2851

Cushing, M. C., \& Vacca, W. D. 2006, AJ, 131, 1797

Dahn, C. C., Harris, H. C., Levine, S. E., et al. 2008, ApJ, 686, 548

Gelino, C. R., Kirkpatrick, J. D., \& Burgasser, A. J. 2008, online database for $670 \mathrm{~L}$ and T dwarfs at DwarfArchives . org (status: 30 September 2008)

Gizis, J. E. 1997, AJ, 113, 806

Gizis, J. E., \& Harvin, J. 2006, AJ, 132, 2372

Hambly, N. C., MacGillivray, H. T., Read, M. A., et al. 2001, MNRAS, 326, 1279

Henry, T. J., Jao, W.-C., Subasavage, et al. 2006, AJ, 132, 2360

Jao, W.-C., Henry, T. J., Beaulieu, T. D., \& Subasavage, J. P. 2008, AJ, 136, 840

Kirkpatrick, J. D. 2005, ARA\&A, 43, 195

Kuiper, G. P. 1939, ApJ, 89, 548

Lépine, S., \& Scholz, R.-D. 2008, ApJ, 681, L33

Lépine, S., Shara, M. M., \& Rich, R. M. 2002, AJ, 124, 1190

Lépine, S., Rich, R. M., \& Shara, M. M. 2003a, AJ, 125, 1598

Lépine, S., Shara, M. M., \& Rich, R. M. 2003b, ApJ, 585, L69 Lépine, S., Rich, R. M., \& Shara, M. M. 2003c, ApJ, 591, L49 Lépine, S., Rich, R. M., \& Shara, M. M. 2007, ApJ, 669, 1235

Monet, D. G., Dahn, C. C., Vrba, F. J., et al. 1992, AJ, 103, 638 Scholz, R.-D., Lehmann, I., Matute, I., \& Zinnecker, H. 2004a, A\&A, 425, 519 Scholz, R.-D., Lodieu, N., \& McCaughrean, M. J. 2004b, A\&A, 428, L25 Sivarani, T., Kembhavi, A. K., \& Gupchup, J. 2004, ApJL, submitted Skrutskie, M. F., Cutri, R. M., Stiening, R., et al. 2006, AJ, 131, 1163 Vrba, F. J., Henden, A. A., Luginbuhl, C. B., et al 2004, AJ, 127, 2948 York, D. G., Adelman, J., Anderson, J. E., et al. 2000, AJ, 120, 1579 